\title{
Downregulation of PIK3CB Involved in Alzheimer's Disease via Apoptosis, Axon Guidance, and FoxO Signaling Pathway
}

\author{
Zhike Zhou ${ }^{1},{ }^{1}$ Jun Bai, ${ }^{2}$ Shanshan Zhong, ${ }^{3}$ Rongwei Zhang, ${ }^{1}$ Kexin Kang, \\ Xiaoqian Zhang, ${ }^{3}$ Ying Xu, ${ }^{2,4}$ Chuansheng Zhao ${ }^{3},{ }^{3}$ and Mei Zhao ${ }^{5}{ }^{5}$ \\ ${ }^{1}$ Department of Geriatrics, The First Affiliated Hospital, China Medical University, Shenyang, 110001 Liaoning, China \\ ${ }^{2}$ Cancer Systems Biology Center, The China-Japan Union Hospital, Jilin University, Changchun, 130033 Jilin, China \\ ${ }^{3}$ Department of Neurology, The First Affiliated Hospital, China Medical University, Shenyang, 110001 Liaoning, China \\ ${ }^{4}$ Computational Systems Biology Lab, Department of Biochemistry and Molecular Biology and Institute of Bioinformatics, \\ The University of Georgia, USA \\ ${ }^{5}$ Department of Cardiology, The Shengjing Affiliated Hospital, China Medical University, Shenyang, 110004 Liaoning, China \\ Correspondence should be addressed to Chuansheng Zhao; cszhao@cmu.edu.cn and Mei Zhao; zhaom1@sj-hospital.org
}

Received 9 June 2021; Accepted 8 January 2022; Published 20 January 2022

Academic Editor: Fabiana Morroni

Copyright (c) 2022 Zhike Zhou et al. This is an open access article distributed under the Creative Commons Attribution License, which permits unrestricted use, distribution, and reproduction in any medium, provided the original work is properly cited.

\begin{abstract}
Objective. To investigate the molecular function of phosphatidylinositol-4,5-bisphosphate 3-kinase catalytic subunit beta (PIK3CB) underlying Alzheimer's disease (AD). Methods. RNA sequencing data were used to filtrate differentially expressed genes (DEGs) in AD/nondementia control and PIK3CB-low/high groups. An unbiased coexpression network was established to evaluate module-trait relationships by using weight gene correlation network analysis (WGCNA). Global regulatory network was constructed to predict the protein-protein interaction. Further cross-talking pathways of PIK3CB were identified by functional enrichment analysis. Results. The mean expression of PIK3CB in AD patients was significantly lower than those in nondementia controls. We identified 2,385 DEGs from 16,790 background genes in AD/control and PIK3CB-low/high groups. Five coexpression modules were established using WGCNA, which participated in apoptosis, axon guidance, long-term potentiation (LTP), regulation of actin cytoskeleton, synaptic vesicle cycle, FoxO, mitogen-activated protein kinase (MAPK), and vascular endothelial growth factor (VEGF) signaling pathways. DEGs with strong relation to AD and low PIK3CB expression were extracted to construct a global regulatory network, in which cross-talking pathways of PIK3CB were identified, such as apoptosis, axon guidance, and FoxO signaling pathway. The occurrence of $\mathrm{AD}$ could be accurately predicted by low PIK3CB based on the area under the curve of $71.7 \%$. Conclusions. These findings highlight downregulated PIK3CB as a potential causative factor of AD, possibly mediated via apoptosis, axon guidance, and FoxO signaling pathway.
\end{abstract}

\section{Introduction}

Alzheimer's disease (AD), referring to an irreversible neurodegenerative disorder, is manifested in cognitive decline, along with behavioral and psychiatric abnormalities of varying extent $[1,2]$. The core hallmarks of AD comprise intracellular hyperphosphorylated tau and extracellular amyloidbeta $(\mathrm{A} \beta)$ plaques, which progressively deteriorate with loss of neurons and synaptic elements [3-7]. Pathologically, $A \beta$ peptides are derived from the continuous cleavage of amyloid precursor protein (APP) by $\beta$ - and $\gamma$-secretases, known as the amyloidogenic pathway related to neurodegeneration
$[8,9]$. This is in competition with the nonamyloidogenic process of $\alpha$-secretase cleaving APP that prevents A $\beta$ formation by releasing soluble amyloid precursor protein alpha $(\operatorname{sAPP} \alpha)[10]$. Imbalance of these two pathways leads to A $\beta$ accumulation, which further elicits early synaptic alterations and ultimately synaptic loss, a process thought to be regulated by phosphoinositide 3 kinase (PI3K) $[11,12]$. Multiple isoforms of PI3K are existed in higher eukaryotes, which can be categorized into three classes (I-III) according to sequence homology and substrate preference [13]. The main function of PI3K is to catalyze the phosphorylation of phosphatidylinositol on the 3-hydroxyl group of the 


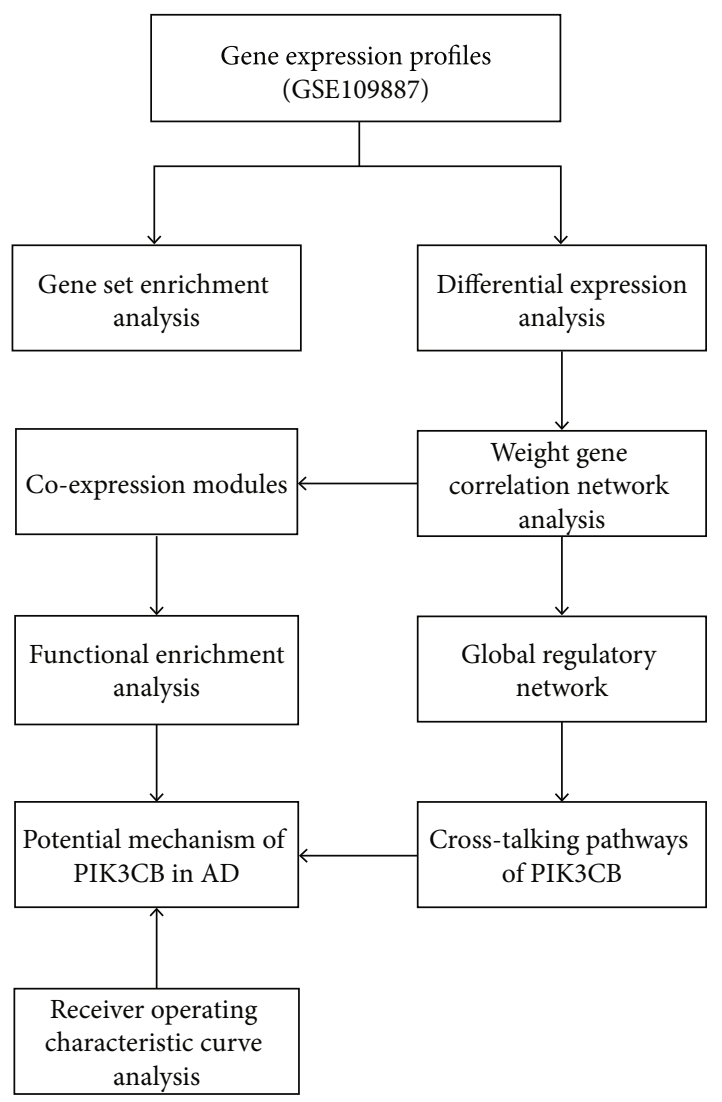

Figure 1: Flow chart of research design. AD: Alzheimer's disease.

inositol ring, successively processed by isoforms from class III, II to I $[14,15]$. Such products of phosphorylated lipids, in turn, provide an anchor for the assembly of downstream proteins that trigger complex intracellular signaling cascades [14]. As an important signaling molecular, dysregulation of PI3K is ubiquitously observed in the development of many diseases, including AD [16]. Despite of no cure presently available for the disease, the exploration of causative factors or crucial regulators (e.g., PI3K) associated with $\mathrm{AD}$ may delay or even prevent the occurrence and progression of $\mathrm{AD}$.

Traditionally, class I PI3K is the most concerned isoform of PI3K family, which consists of a regulatory subunit and a catalytic subunit that play pivotal roles in cell proliferation, differentiation, and survival (or apoptosis) [17-19]. In terms of the catalytic subunit of class I PI3K, phosphatidylinositol4,5-bisphosphate 3-kinase catalytic subunit beta (PIK3CB) is highly expressed in neurons implicated in synapse formation and cell cycle regulation $[20,21]$. Several lines of evidence have demonstrated that PI3K signaling pathways are linked to the pathophysiology of AD. For instance, TREM2 alleviates neuroinflammation and cognitive impairment through $\mathrm{PI} 3 \mathrm{~K} / \mathrm{AKT} / \mathrm{FoxO}$ a signaling in $\mathrm{AD}$ mice; conversely, this neuroprotective effect of TREM2 can be eliminated with PI3K inhibitors [22]. Similar results are also replicated by sulforaphene administration, which attenuates neuroinflammatory response and hyperphosphorylation of tau via modulating the PI3K/Akt/GSK-3 $\beta$ pathway [23]. To the best of our knowledge, the pathogenic mechanism whereby dysreg- ulation of PIK3CB mediates AD is not well understood. Toward this, we sought to conduct a comprehensive genomic analysis on basis of gene expression profile and functional annotations [24], which might shed light on the molecular role of PIK3CB in the pathogenesis of $\mathrm{AD}$.

\section{Materials and Methods}

2.1. Data Resources. Microarray RNA sequencing (RNA-seq) data from middle temporal gyrus of 78 human samples (46 AD patients and 32 age-matched nondementia controls) were accessible through Gene Expression Omnibus (GEO) Series accession number GSE109887 [25, 26]. Illumina HumanHT-12 V4.0 expression beadchip was used to detect the expression of 16,790 annotated genes with 31,700 probes. To reduce prediction error on cross studies, the normalization of gene expression profiles was processed with the normalizeBetweenArrays function in R package [27].

2.2. Gene Set Enrichment Analysis (GSEA). GSEA is a widely used computational method assessing whether enrichment of a predefined gene set is statistically significant between two biological states [28]. During the process of functional annotation, 1000 permutations were set up to screen biological processes (BP) of gene ontology (GO) terms utilizing ClusterProfler, enrichplot and GSEABase packages [29]. The visualization of GSEA data was accomplished by ggplot2 package. A pvaluecutoff of 0.05 was determined as the screening threshold for significant enrichment.

2.3. Differential Expression Analysis. Taken the average expression value of PIK3CB to be the boundary, enrolled samples were dichotomized into PIK3CB-low and PIK3CB-high cohort. Using lmFit and eBayes functions, we identified the differences of gene expression in $\mathrm{AD} /$ control and PIK3CB-low/high groups, respectively [30]. A fold change $(\mathrm{FC})>1.7$ combined with a false discovery rate (FDR)-adjusted $p$ cutoff of $<0.05$ was adopted to define differentially expressed genes (DEGs) [31-33]. Analysis of twodimensional hierarchical cluster was carried out by $\mathrm{R}$ software of limma package [32]. Volcano plot and heat map were employed to visualize the expression of DEGs in the screening and cluster analyses.

2.4. Coexpression Network Analysis. Following the default parameters of weight gene correlation network analysis (WGCNA), overlapping DEGs between $\mathrm{AD} /$ control and PIK3CB-low/high cohorts were disposed to create an unbiased coexpression network. The preponderance of WGCNA is that it converts intricate microarray data into gene coexpressed modules, providing insight into a signal network that may be associated with phenotypic traits of interest [34]. To ensure the reliability of network outcomes, sample clustering diagram was plotted to eliminate outliers with low inter-array correlation using hclust function. An appropriate soft-thresholding power of 12 was selected with pickSoftThreshold function to achieve the integral connectivity of gene coexpression modules, so that the network utmost approaches the authentic biological state [35]. Module eigengene (ME) is the first major element for a module, 


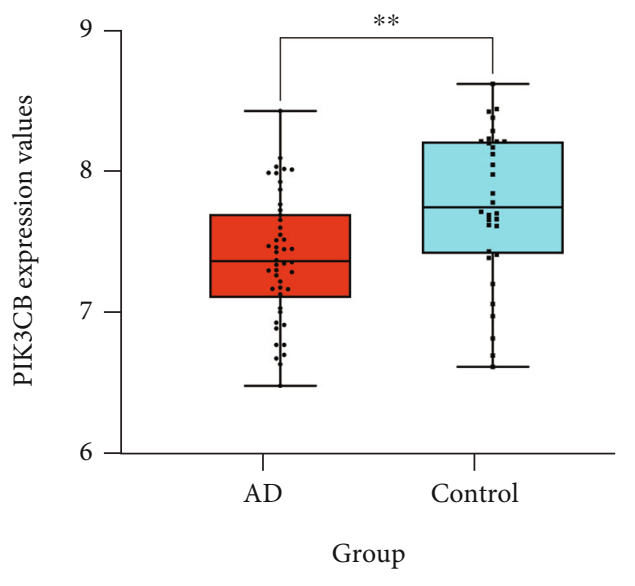

$\mathrm{AD}$

Control

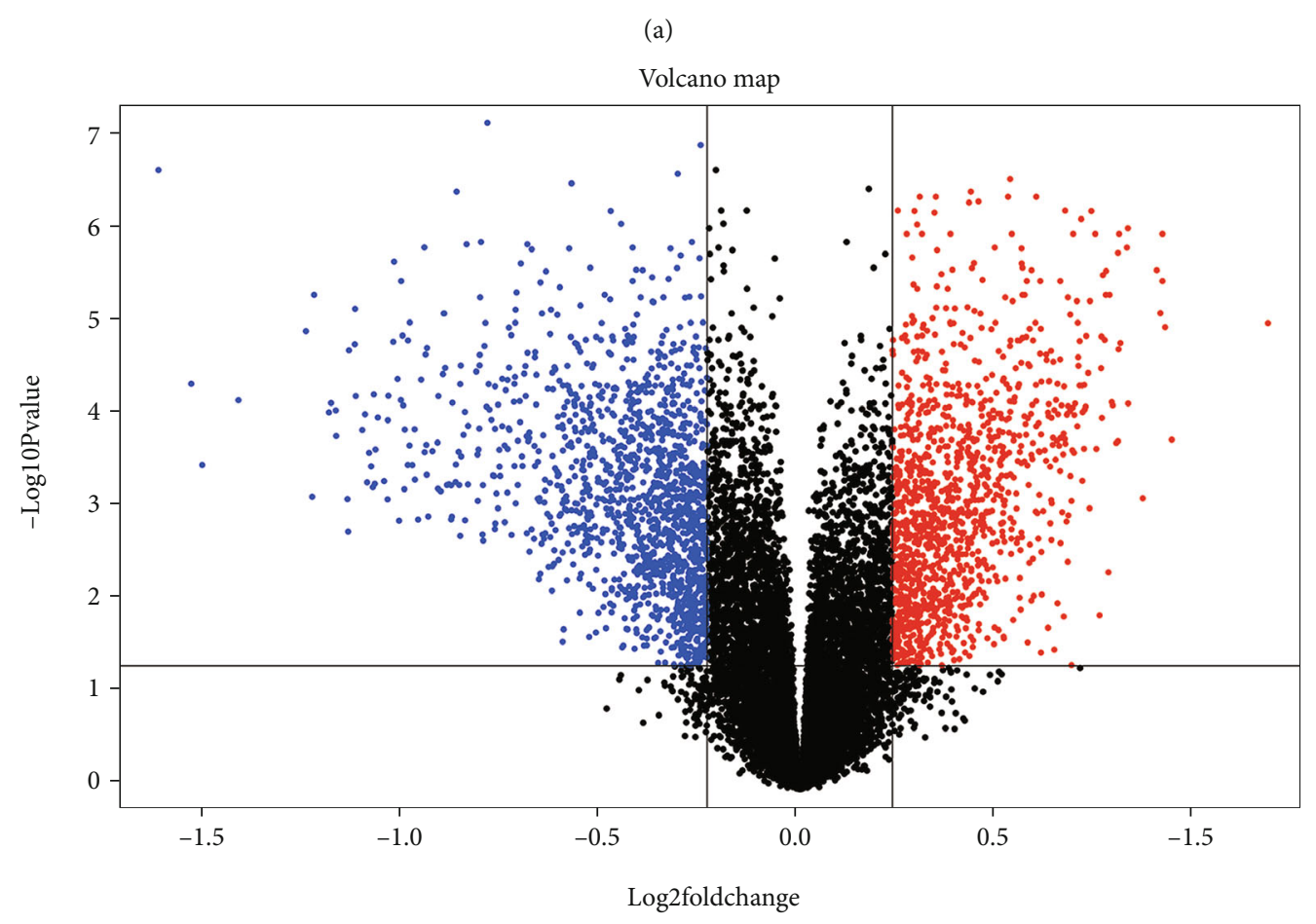

(b)

Figure 2: Continued. 


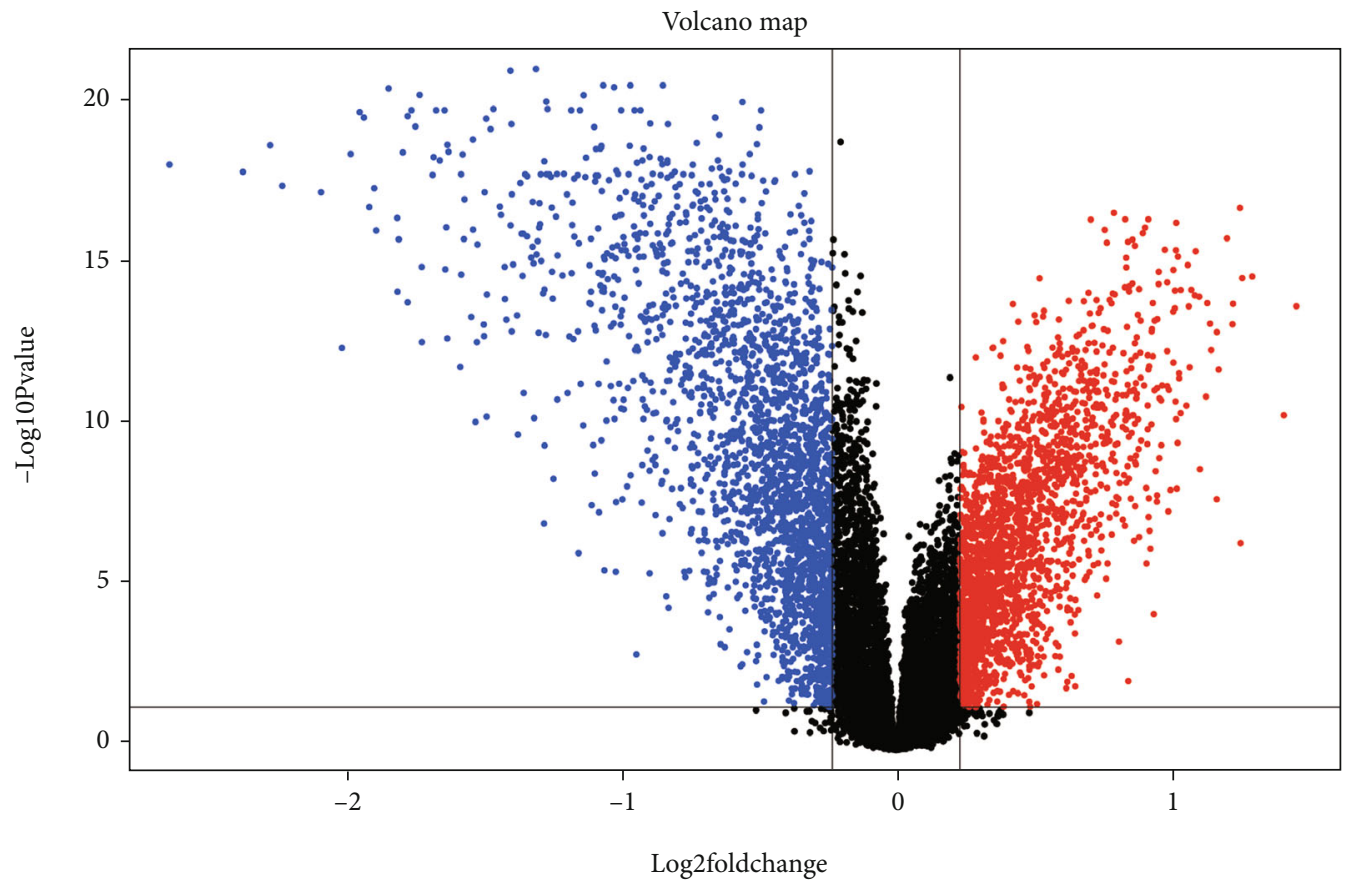

(c)

Figure 2: Continued. 


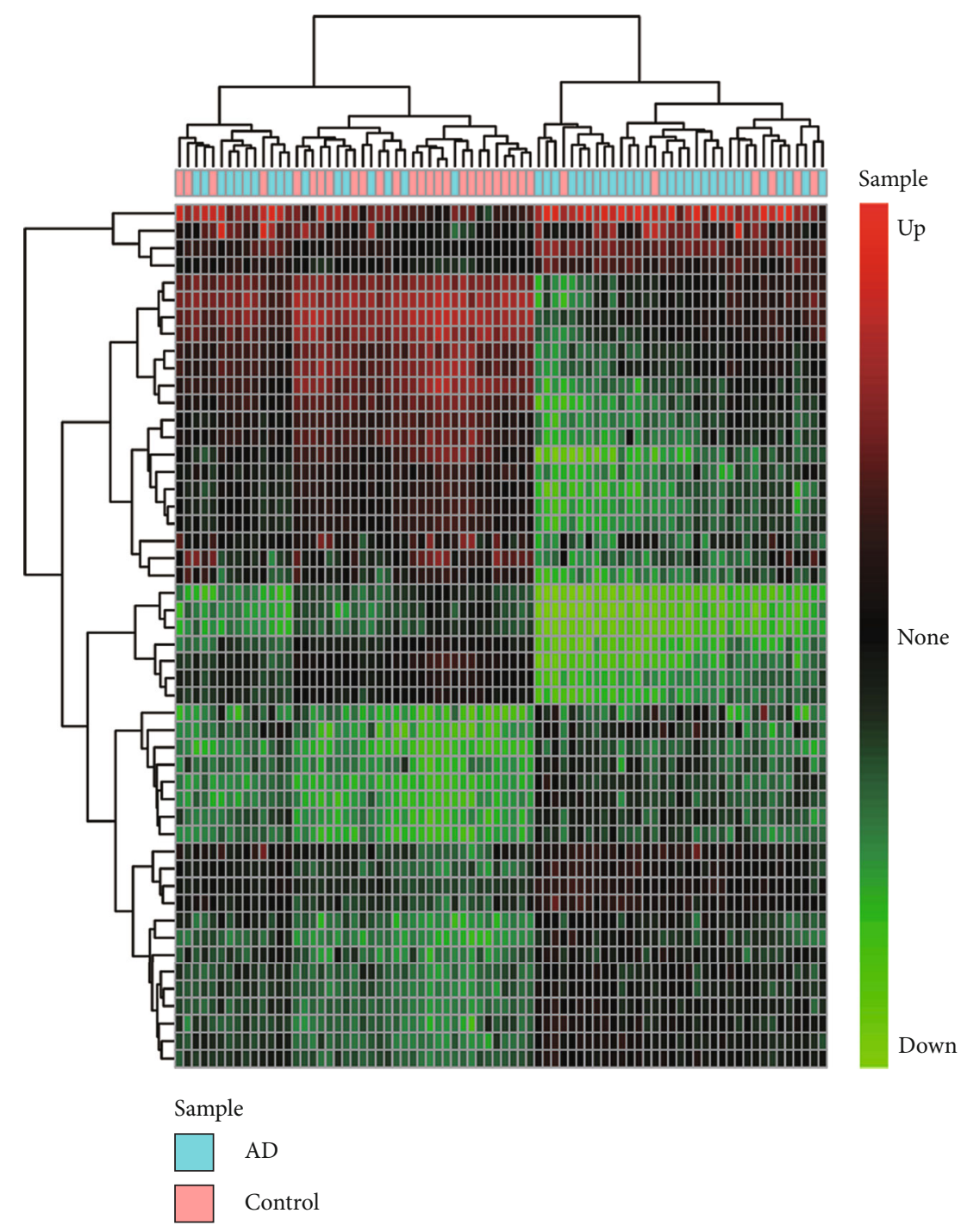

(d)

Figure 2: Differential expression analysis. (a) PIK3CB expression between AD and nondementia controls. ((b) and (c)) Volcano plots of DEGs in $\mathrm{AD} /$ control and PIK3CB-low/high cohorts: red indicates upregulated, while blue represents downregulated. (d) Heatmap of the first 25 up- and downregulated DEGs: red to green indicates gene expression alterations from upregulated to downregulated. AD: Alzheimer's disease; DEGs: differentially expressed genes.

known as the module representative that accounts for the maximum possible variability of all genes in a module [36]. Correlation coefficients of all genes with each ME were calculated to construct a hierarchical clustering tree, by which branches of $>30$ genes were reassembled into coexpression modules with unique color tags $[37,38]$. Functional enrichment analyses were performed to filtrate Kyoto Encyclopedia of Genes and Genomes (KEGG) pathways using clusterProfiler package.

2.5. Global Regulatory Network and Cross-Talking Pathways of PIK3CB. Genetic phenotype and intramodular connectivity were measured by gene significance (GS) and module membership (MM), respectively, the relationships of which were plotted in a scatter diagram adopting verboseScatterplot function [39]. Based on an online database of Search Tool for the Retrieval of Interacting Genes (STRING, http:// www.stringdb.org/), the interaction between DEGs was explored by establishment of protein-protein interaction (PPI) network [40]. The cytoscape software was used to visualize the global regulatory network, as well as the crosstalking pathways of PIK3CB that were enriched by pathway enrichment analyses [41, 42].

2.6. Analysis of Receiver Operating Characteristic Curve (ROC). ROC analysis was conducted to estimate the classifier performance of sequential output, including sensitivity and specificity parameters, as measured by the area under the curve (AUC) [43, 44]. Diagnostic performance of PIK3CB to distinguish $\mathrm{AD}$ cases from nondementia controls was assessed using $p R O C$ package in R. An AUC value of $100 \%$ indicated complete prediction, while $50 \%$ represented random selection. 


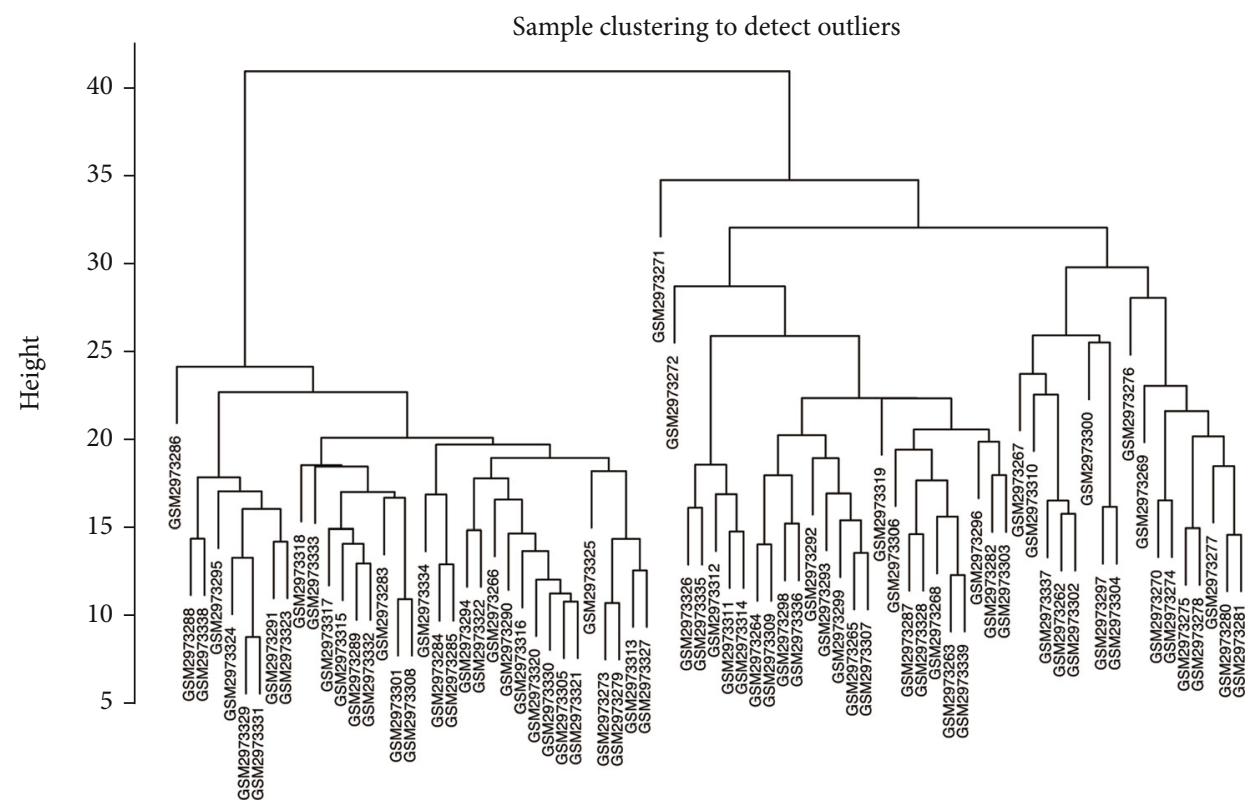

(a)

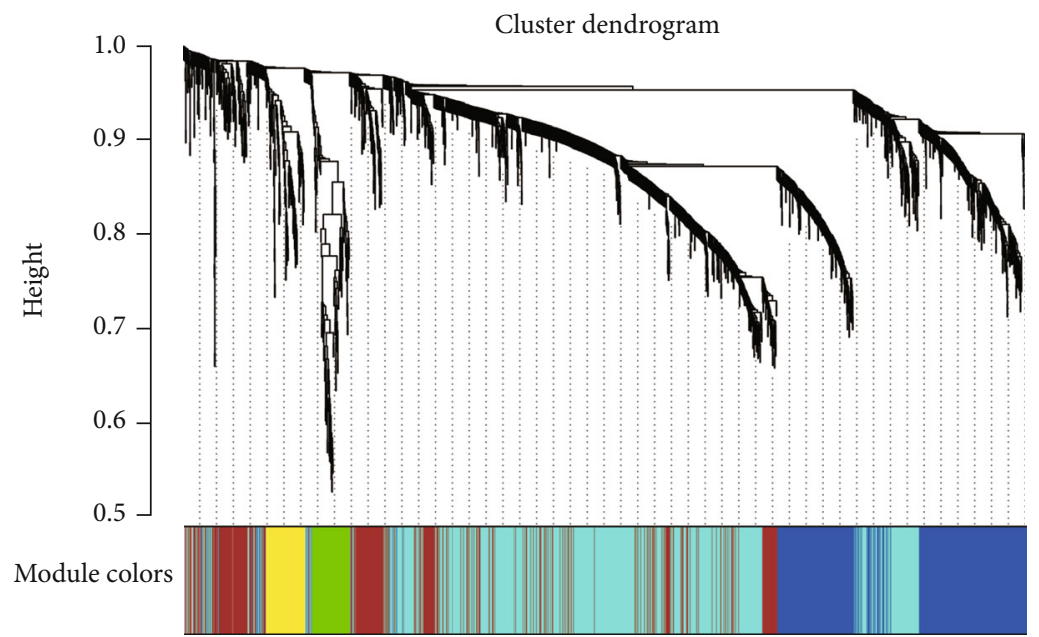

(b)

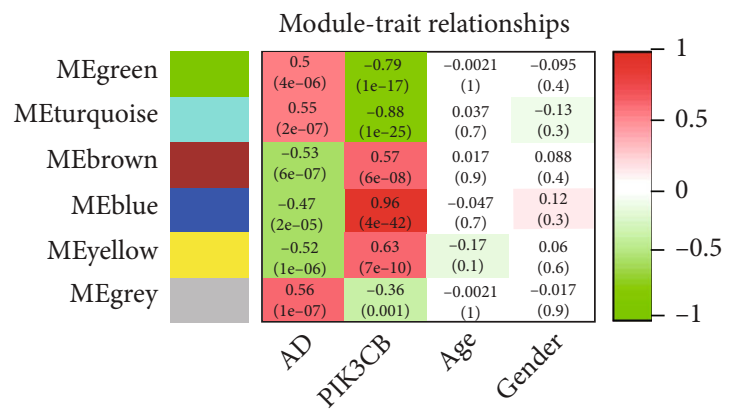

(c)

Figure 3: Continued. 


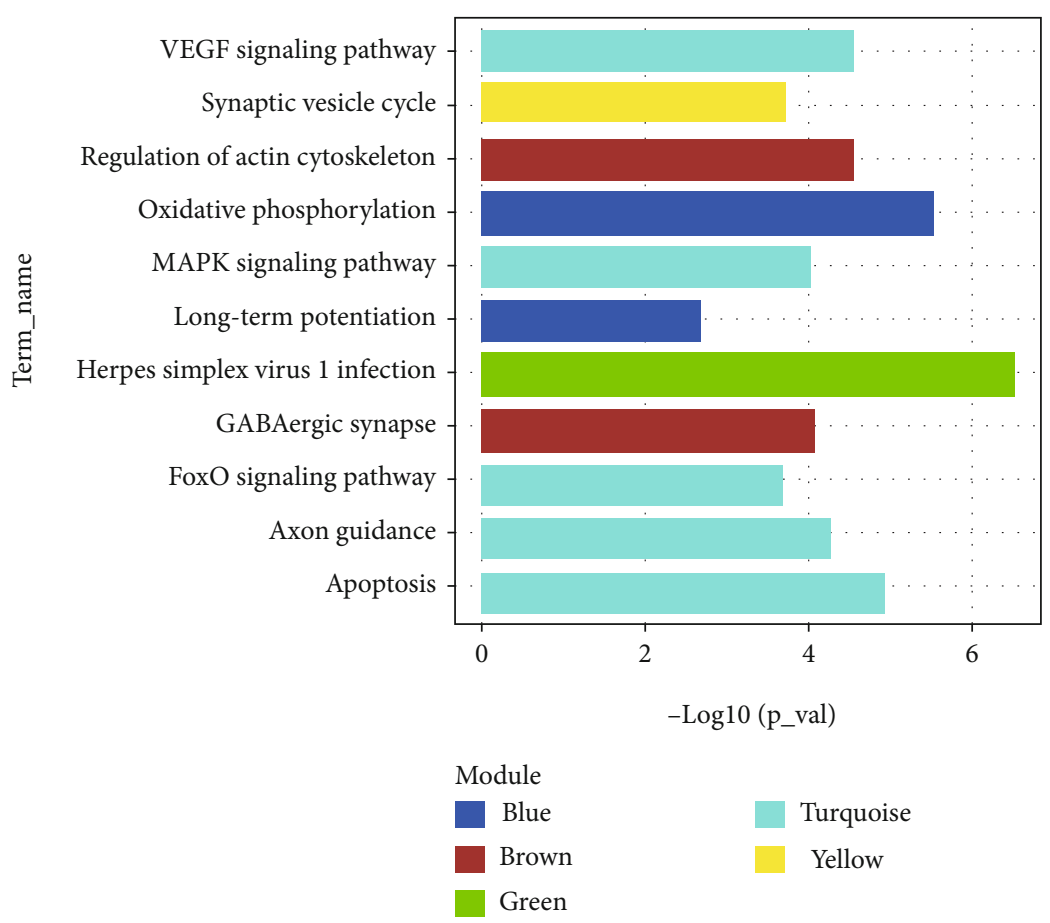

(d)

Figure 3: Weighted correlation network analysis. (a) Sample clustering to detect outlines. (b) Dendrogram of genes clustered by different module colors: gray represents noncoexpression genes. (c) Module-trait relationships: red to green indicates a positive to negative correlation of module eigengenes with phenotypes. (d) KEGG enrichment results of coexpression modules. AD: Alzheimer's disease; KEGG: Kyoto Encyclopedia of Genes and Genomes.

2.7. Statistical Analysis. Continuous variables between AD patients and nondementia controls were compared by applying $t$ test or the nonparametric Mann-Whitney $U$ test. During the process of WGCNA analysis, the statistical relationship between gene coexpressed modules and phenotypic traits of interest was estimated by Pearson correlation coefficient (PCC) analysis. Using the approach of Hanley-McNeil test, predictive accuracy of PIK3CB was verified by ROC analysis, the result of which was quantified by AUC value. Two-tailed $p$ values of $<0.05$ were defined as the standard of statistical significance. All statistical analyses were conducted by employing GraphPad Prism software (version 8.3.1) and R package (version 3.6.2).

\section{Results}

3.1. Overall Research Design. The workflow diagram of this research was detailed in Figure 1. Herein, the GSE109887 dataset in GEO database was selected for bioinformatic mining. Subsequently, an integrative method of differentially expression analysis and clinical phenotype-based WGCNA was employed to build AD-related gene coexpression modules by a comparison between $\mathrm{AD}$ and nondementia controls. The cellular processes of each module were further enriched by functional enrichment analysis, which provided an understanding of the biological functions of coexpressed genes at the cellular level. Thenceforth, we constructed a global regulatory network based on module genes strongly interacting with $\mathrm{AD}$ and $\mathrm{PIK} 3 \mathrm{CB}$, wherein the cross- talking pathways of PIK3CB were identified. Additionally, diagnostic performance of $\mathrm{PIK} 3 \mathrm{CB}$ in $\mathrm{AD}$ prediction was validated by ROC analysis.

3.2. Identification of Differentially Expressed Genes. Compared with controls, the mean gene expression of PIK3CB was significantly downregulated in $A D$ patients $(7.38 \pm 0.44$ versus $7.76 \pm 0.53 ; p<0.01$ ) (Figure 2(a)). Through preliminary processing of microarray data, 16,790 background genes were generated for further DEG identification. Significant differences in the expression of 2,675 genes (1,616 up- and 1,850 downregulated) were screened in $\mathrm{AD}$ relative to nondementia cohort (Figure 2(b)), while 4,393 genes (2,096 up- and 2,297 downregulated) were differentially expressed in PIK3CB-low versus high group (Figure 2(c)). Subsequently, a total of 2,385 overlapping DEGs (1,093 up- and 1,292 downregulated) were filtrated between $\mathrm{AD} /$ control and PIK3CB-low/high groups. The expression of the first 25 up- and downregulated DEGs in $\mathrm{AD}$ versus nondementia controls was shown in heat map (Figure 2(d)), which exhibited substantially different biology between these two groups.

3.3. Coexpression Modules and Functional Enrichment Analysis. During the process of hierarchical clustering detection of outliers by using average linkage, all samples passed the cut-off line (height $=45$ ) and could be enrolled in subsequent gene coexpression network analysis (Figure 3(a)). As shown in Figure 3(b), coexpressed genes were clustered into 


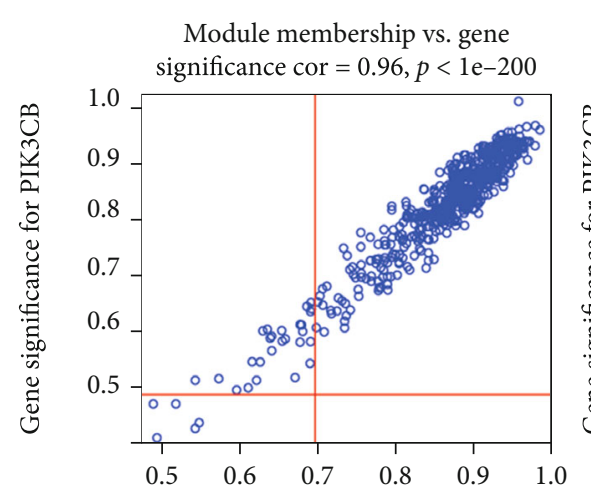

Module membership in blue module

Module membership vs.gene significance cor $=-0.62, p=0.042$

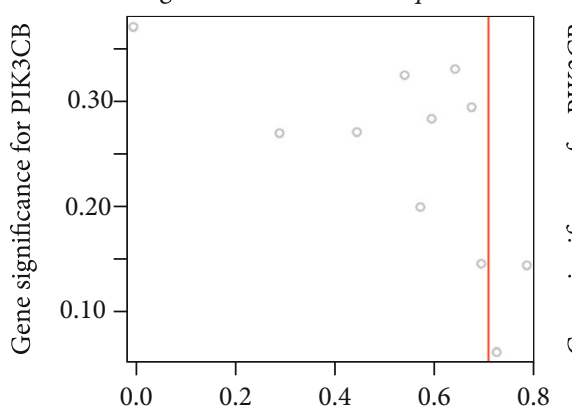

Module membership in grey module

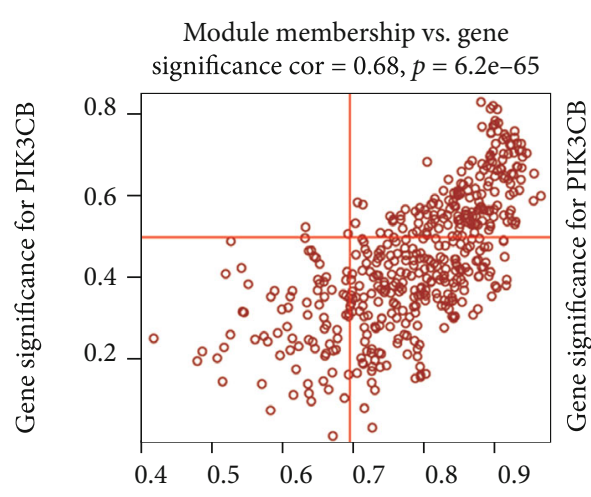

Module membership in brown module

Module membership vs.gene significance cor $=0.81, p<1 \mathrm{e}-200$

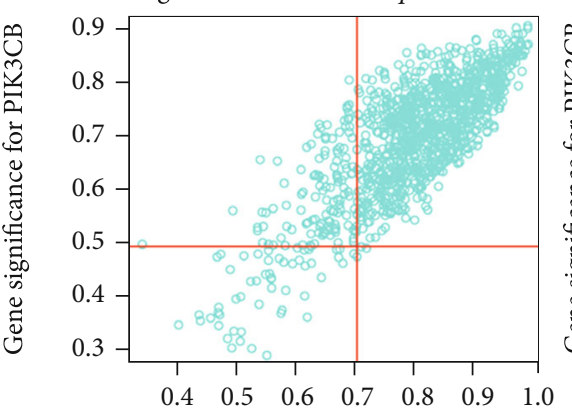

Module membership in turquoise module

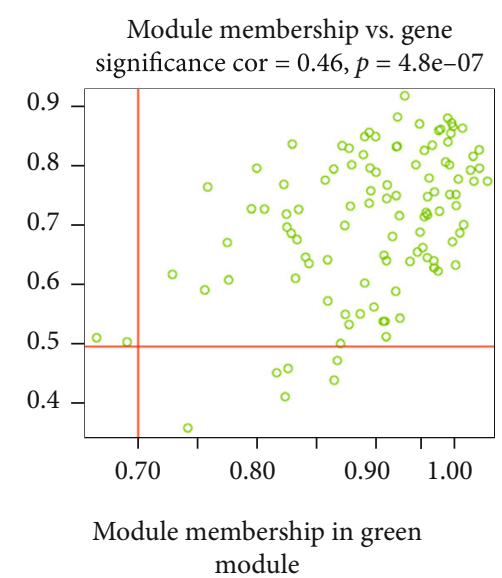

Module membership vs.gene significance cor $=-0.62, p=0.042$

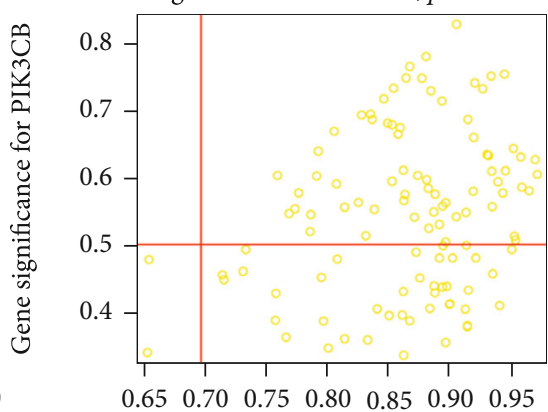

Module membership in yellow module

(a)

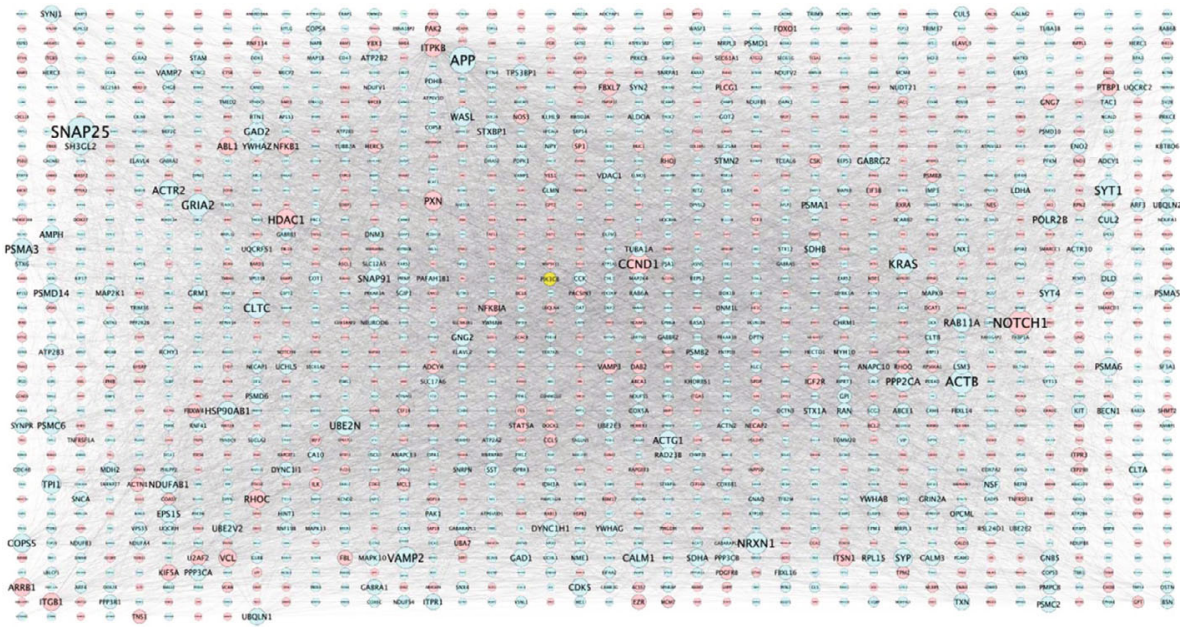

(b)

Figure 4: Continued. 


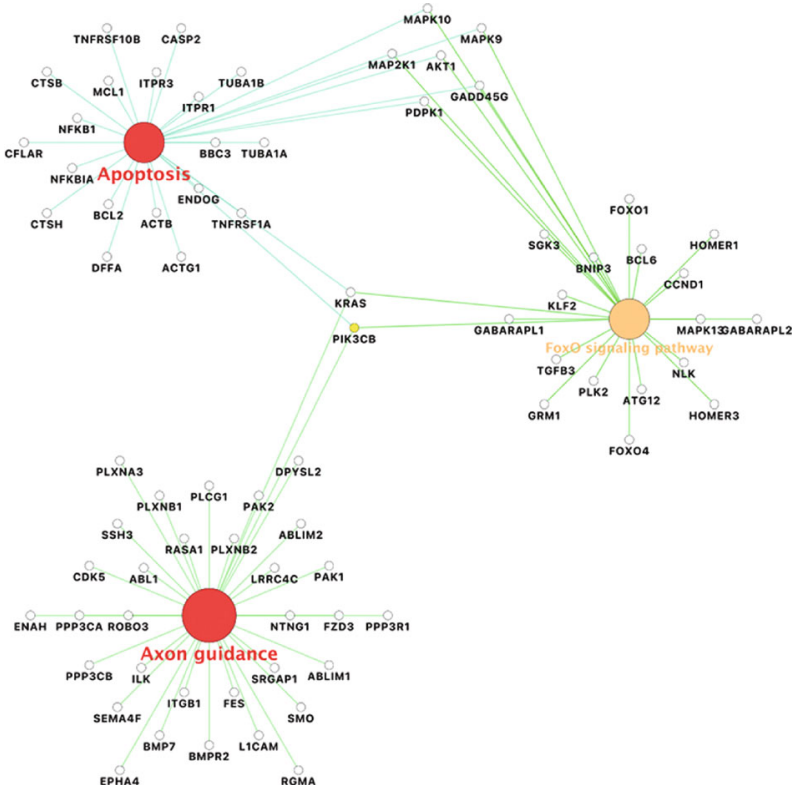

(c)

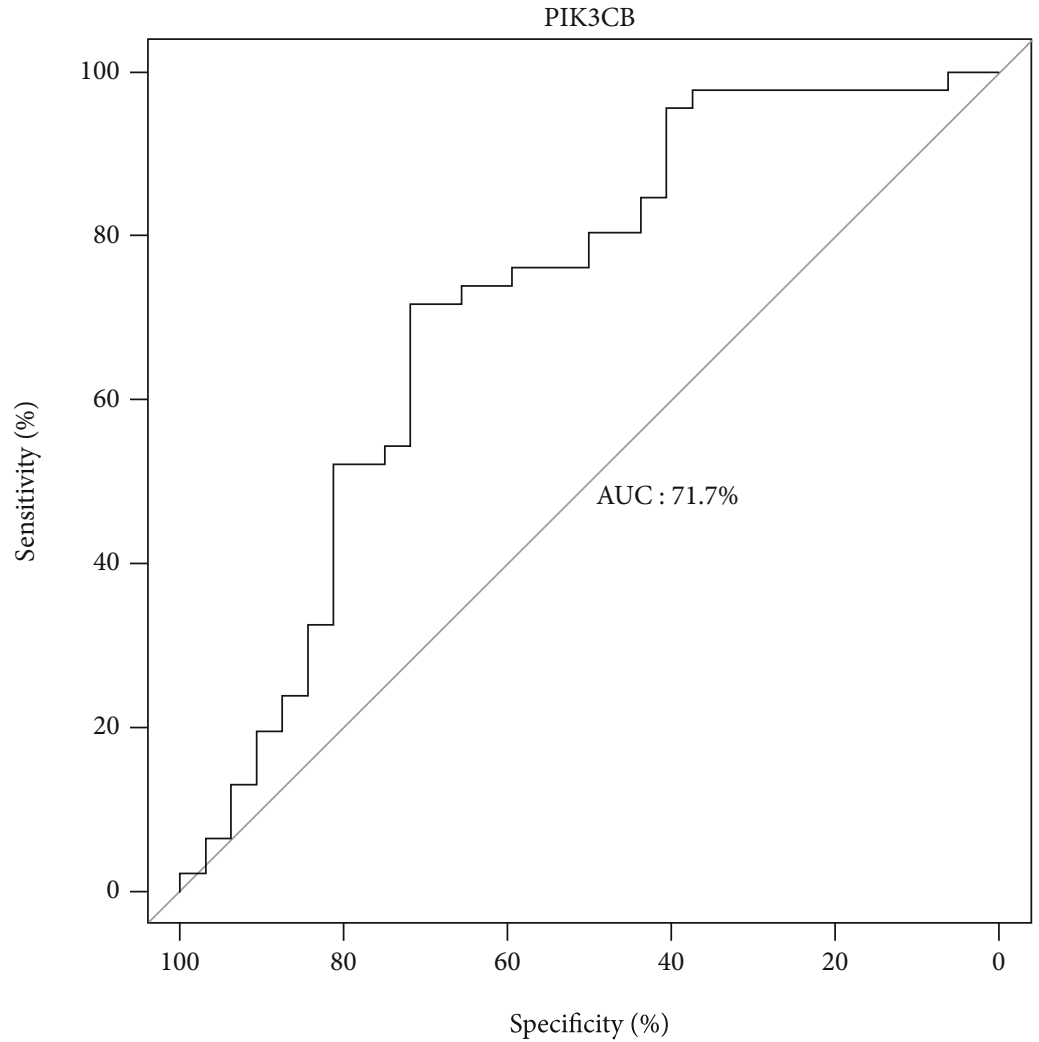

(d)

FIGURE 4: Module-pathway regulatory network and ROC analysis. (a) Scatter plot of module membership related to gene significance. (b) Global regulatory network based on PIK3CB-interacting genes: node size reflects the connectivity between genes; red indicates upregulated; blue and yellow represent downregulated. (c) Cross-talking pathways of PIK3CB: yellow is the downregulated PIK3CB. (d) AUC value of PIK3CB in predicting AD onset. AD: Alzheimer's disease; AUC: area under the curve; ROC: receiver operating characteristic curve.

five coexpression modules by WGGNA, implying that genes in each cluster were transcriptionally correlated; whereas the remaining noncoexpressed genes were grouped into a nonfunctional module, namely, the gray module. Heatmap of module-trait relationships (Figure 3(c)) revealed that green and turquoise modules had the significantly positive correlation with $\mathrm{AD}$ (green: correlation coefficient $=0.5, p=4 e-06$ ; turquoise: correlation coefficient $=0.55, p=2 e-07)$ and 


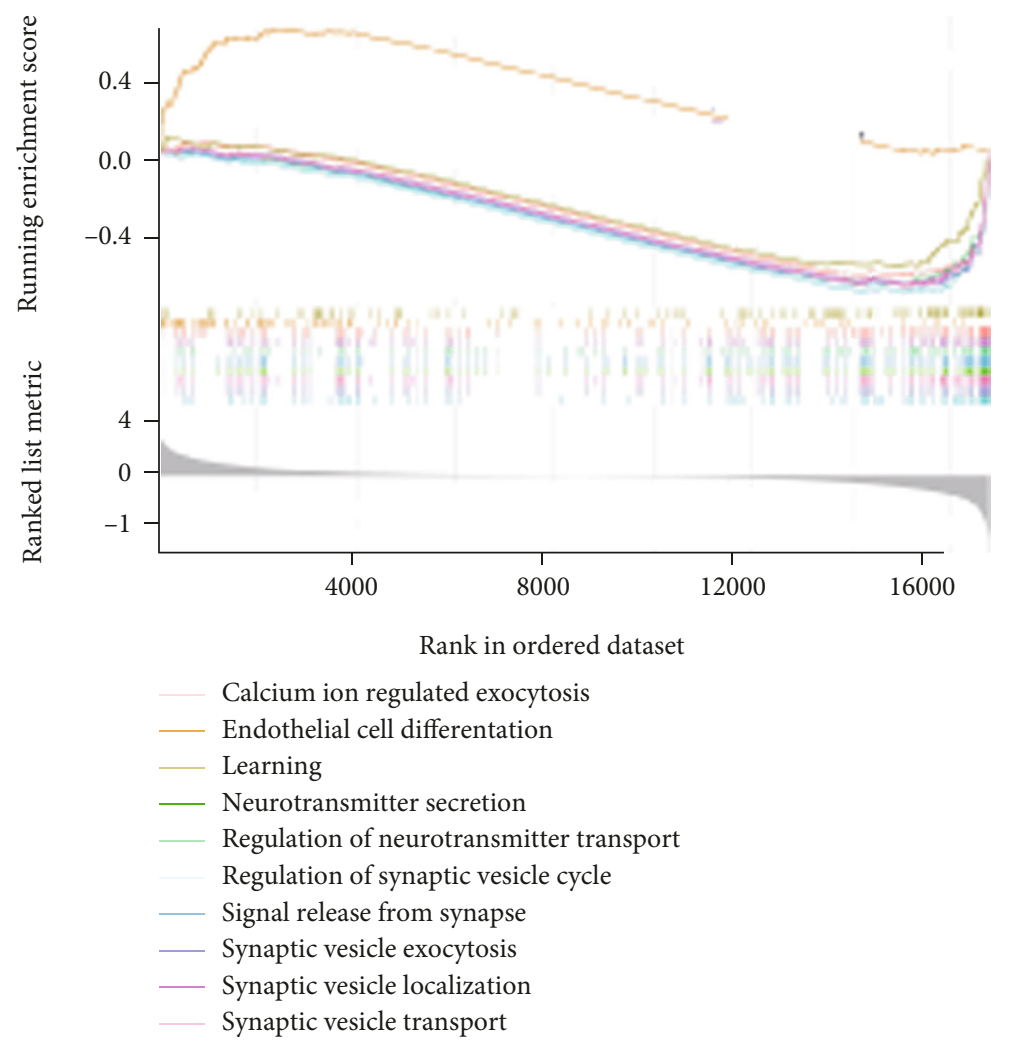

(a)

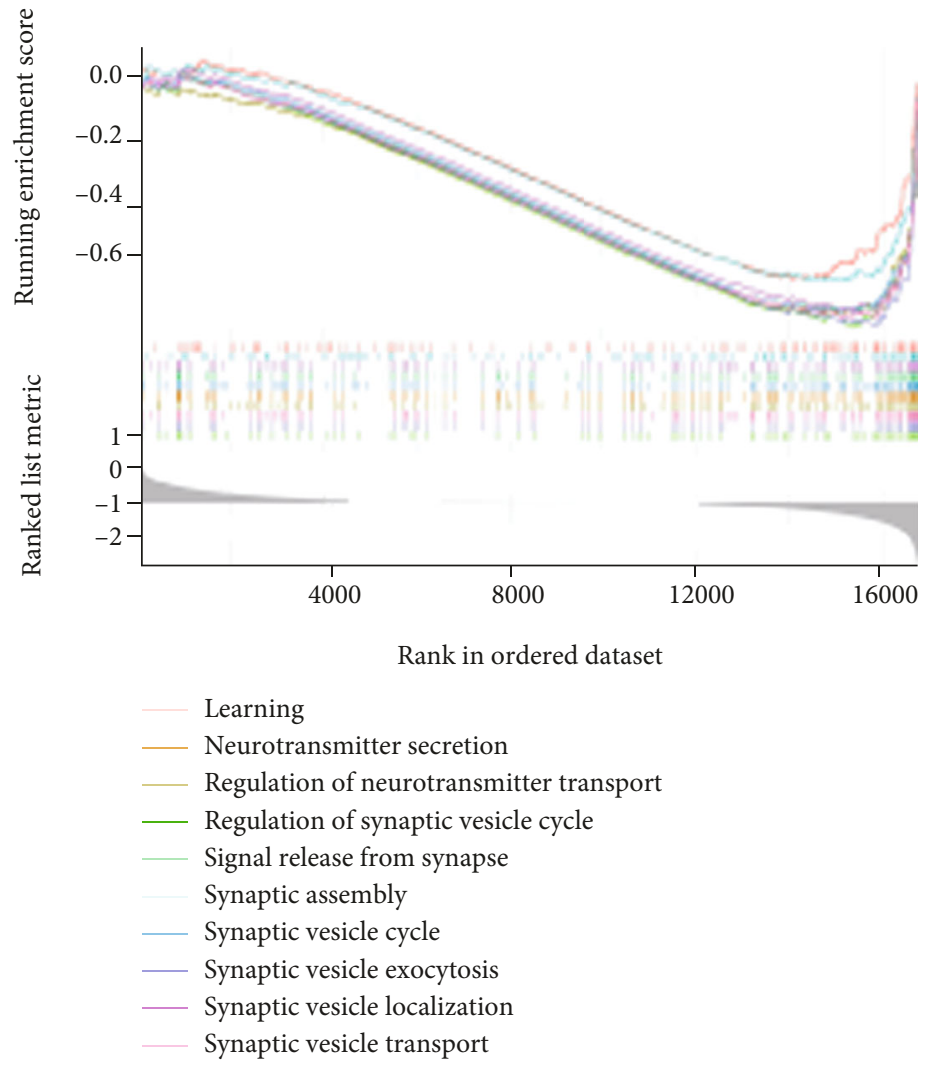

(b)

Figure 5: Gene set enrichment analysis. (a) Gene functional annotation of biological processes in AD. (b) Gene functional annotation of biological processes in PIK3CB-low group. AD: Alzheimer's disease. 
negative correlation with PIK3CB expression (green: correlation coefficient $=-0.79, \quad p=1 e-17$; turquoise: correlation coefficient $=-0.88, p=1 e-25)$; while brown, blue, and yellow modules were negatively correlated with $\mathrm{AD}$ (brown: correlation coefficient $=-0.53, p=6 e-07$; blue: correlation coefficient $=-0.47, \quad p=2 e-05 ; \quad$ yellow: correlation coefficient $=-0.52, p=1 e-06)$ and positively relevant to $\mathrm{PIK} 3 \mathrm{CB}$ expression (brown: correlation coefficient $=0.57, p=6 e-08$; blue: correlation coefficient $=$ $0.96, p=4 e-42$; yellow: correlation coefficient $=0.63, p=7$ $e-10)$. Functional enrichment analysis of KEGG pathways (Figure 4(d)) showed that DEGs in blue module were enriched in oxidative phosphorylation and long-term potentiation (LTP); DGEs of brown module were involved in GABAergic synapse and regulation of actin cytoskeleton; DEGs in green module participated in herpes simplex virus 1 infection; DEGs of turquoise module were enriched in apoptosis, axon guidance, FoxO, mitogen-activated protein kinase (MAPK), and vascular endothelial growth factor (VEGF) signaling pathways; DEGs in yellow module were involved in synaptic vesicle cycle. Accordingly, the specific mechanistic pathways of coexpressed genes in each module in $\mathrm{AD}$ were functionally screened out.

3.4. Module-Pathway Regulatory Network and ROC Analysis. As shown in Figure 4(a), scatter diagram between MM and GS exhibited a significant correlation between intramodular connectivity and genetic phenotypes in the coexpression modules (blue: correlation coefficient $=0.96, p<1 e-200$; brown: correlation coefficient $=0.68, p=6.2 e-65$; green: correlation coefficient $=0.46, \quad p=4.8 e-07$; turquoise: correlation coefficient $=0.81, \quad p<1 e-200 ; \quad$ yellow: correlation coefficient $=0.25, p=0.0079$ ), suggesting that the five coexpression modules were prominently associated with low expression of PIK3CB in AD patients. To accurately identify the mechanistic pathways of $\mathrm{PIK} 3 \mathrm{CB}$ in $\mathrm{AD}$ onset, DEGs strongly interacting with PIK3CB (meeting the inclusion criteria of $\mathrm{MM}>0.7$ and $\mathrm{GS}>0.5$ ) were extracted to construct a global regulation network (Figure 4(b)) based on STRING database. Using pathway enrichment analyses, further cross-talking pathways of PIK3CB, such as apoptosis, axon guidance, and FoxO signaling pathway, were determined in the network (Figure 4(c)). The ROC analysis (AUC $=71.7 \%$ ) showed that low PIK3CB performed a good performance in differentiating $\mathrm{AD}$ cases from nondementia controls (Figure 4(d)).

3.5. GESA Validation in Biological Processes. To validate the potential functional mechanisms involved by $\mathrm{PIK} 3 \mathrm{CB}$ in $\mathrm{AD}, \mathrm{GSEA}$ was performed for BP enrichment according to a predefined gene set. The enriched BP in AD cohort (Figure 5(a)) was primarily linked to calcium ion regulated exocytosis, endothelial cell differentiation, learning, neurotransmitter secretion, signal release form synapse, and synaptic vesicle transport. The functions of core enrichment genes in the PIK3CB-low group (Figure 5(b)) were mainly distributed in BP of learning, synapse assembly, synaptic vesicle cycle, signal release from synapse, neurotransmitter secretion, and synaptic vesicle transport. These data under- lined that low PIK3CB expression might contribute to the pathogenesis of $\mathrm{AD}$ through biological processes related to learning, neurotransmitter secretion, signal release form synapse, and synaptic vesicle transport.

\section{Discussion}

To improve our understanding of the AD phenotype modified by PIK3CB, we took advantage of the RNA-seq data to screen differentially expressed genes between $\mathrm{AD}$ and nondementia controls for integrative genomic analysis. The results of GSEA showed that functions of core enrichment genes were distributed in the BP of learning, neurotransmitter secretion, signal release form synapse, and synaptic vesicle transport. Historically, neurotransmitters have been described as the fundamental neurochemicals of signaling between presynaptic and postsynaptic neurons, responsible for the maintenance of synaptic and cognitive function [45-48]. Of particular note was that these biological processes were significantly associated with $\mathrm{AD}$ and low PIK3CB expression, indicating an important role of PIK3CB in $\mathrm{AD}$ pathogenesis. Consequently, this promoted us to construct a global regulatory network and coexpression modules of DEGs interacting with PIK3CB to illuminate the genomic mechanism of PIK3CB in the development of $\mathrm{AD}$.

The findings emerging from WGCNA revealed that DEGs of coexpression modules were significantly correlated with $\mathrm{AD}$ and $\mathrm{PIK} 3 \mathrm{CB}$ expression, which were enriched in apoptosis, axon guidance, LTP, synaptic vesicle cycle, FoxO, MAPK, and VEGF signaling pathways. There is evidence that APP aggregates in axonal growth cones, acting as a coreceptor for axon guidance and cell migration cues through its interaction with the extracellular matrix [49-51]. More specifically, $\operatorname{sAPP} \alpha$-the secreted product of $\alpha$-secretase APP cleavage-antagonizes an inhibitor of axon guidance cue termed Sema3A, contributing to cell movement and axon outgrowth [52]. As supported by evidence in neural stem cell-derived neurons, inhibition of $\operatorname{sAPP} \alpha$ secretion abolishes depolarization-induced neurite outgrowth and elongation [53]. Furthermore, sAPP $\beta$-the secreted product of $\beta$-secretase APP cleavage-not only promotes rapid neural differentiation but also loosens intercellular adhesion, which is thought to be critical for axonal outgrowth [54-56]. In addition, $A \beta$-the secreted product of $\beta$ - and $\gamma$-secretase APP cleavage-is the major culprit of AD that impedes axon outgrowth by inducing allosteric collapse of growth cone, giving rise to impaired cognitive recovery $[57,58]$. Altogether, the evidence presented above strongly points to a linkage between axon guidance and AD neurodegeneration. Axon guidance molecules including nerve growth factor (NGF) and insulin-like growth factor (IGF1) have been shown to control axonal growth, a process that relies on tight regulation and localized activation of PI3K [59]. It has long been recognized that PI3K can both stabilize polymerized microtubules and interfere with microtubule polymerization, thus, modulating microtubule dynamics prerequisite for NGF-induced axon elongation [60]. Coincident outcomes have also been reported in an in vitro model of a biocompatible guidance device where a linear 
propagation of IGF-1 gradients sequentially directs axon outgrowth [61]. Notably, an essential step in the initiation of axonal outgrowth is precisely the activation of PI3K by IGF-1 and its receptors [62, 63]. By contrary, inhibition of $\mathrm{PI} 3 \mathrm{~K}$ enhances the response to growth cone collapse, which in turn hinders axon elongation $[64,65]$, consistent with our findings of low PIK3CB-mediated AD pathogenesis involving axon guidance.

An extensive array of studies support that apoptosis underlies the pathogenic mechanisms of neuronal cell death in AD. Analytic results of postmortem brain tissues provided evidence for a 50-fold increase of apoptosis in $\mathrm{AD}$ patients relative to nondementia controls [66]. As the most prevalent genetic risk factor for $\mathrm{AD}$, apolipoprotein $\mathrm{E} 4$ (apoE4) has been identified to trigger apoptosis, leading to a series of detrimental consequences such as impaired neuroplasticity and cognitive decline [67]. This is confirmed by in vitro cell cultures that apoE4 induces an increase of apoptotic cell death in a subtype-specific manner $[8,68$, 69]. Additionally, Takuma et al. found that mitochondrial dysfunction and endoplasmic reticulum- (ER-) induced stress had implications in the execution of apoptosis relevant to $\mathrm{AD}[70]$. One plausible interpretation is that $\mathrm{A} \beta$ depletes $\mathrm{ER} \mathrm{Ca}^{2+}$ reserves to promote excessive uptake of $\mathrm{Ca}^{2+}$ into mitochondria, causing cytosolic $\mathrm{Ca}^{2+}$ overload and thus to activate the mitochondrial-mediated apoptosis [71]. On the other hand, a growing body of research has directly or indirectly linked PIK3CB to apoptosis. In the rat model of subarachnoid hemorrhage, ErbB4-induced activation of PIK3CB increased yes-associated protein (YAP) expression, a terminal effector of Hippo signaling that dramatically improved neurological deficits and apoptosis; meanwhile, inhibition of ErbB4 or YAP knockdown could eliminate this anti-apoptotic effect [72]. Likewise, subsequent in vitro experiment on human glioma cell lines demonstrated that inhibition of PIK3CB by AZD6482 induced apoptosis and cell cycle arrest, as detected using flow cytometry with propidium iodide staining [18]. In fact, PIK3CB has attracted considerable attention as a selective survival factor for cancer therapy based on its critical role in apoptosis [73-76].

Taking into consideration that $\mathrm{A} \beta$ increases the production of reactive oxygen species (ROS), AD may be at higher risk due to mitochondrial oxidative stress through FoxO signaling pathway $[77,78]$. Upregulation of FoxO transcriptional activity has been shown to drive the expression of genes related to antioxidative response by inducing epigenetic modification, contributing to intracellular metabolic homeostasis and oxidative stress clearance [79]. This activation, however, is supposed to be moderate rather than sustained, since the latter mode yields apoptosis instead of resistance to oxidative stress [80-82]. During the progression of $\mathrm{AD}$, inhibition of FoxO disrupts mitochondrial energy metabolism through excessive release of ROS, resulting in impaired synaptic transmission and neuronal apoptosis $[83,84]$. As supported by an in vitro experiment from animal $\mathrm{AD}$ models, $\mathrm{A} \beta$-induced Ros activates p66Shc, an adaptor protein that triggers phosphorylation (i.e., inactivation) of FoxO, which aggravates the accumulation of oxidative stress and thus to neuronal and synaptic loss [85].
Relatedly, in response to oxidative stress stimuli, PI3K activates a downstream serine/threonine kinase, termed as protein kinase $\mathrm{B}(\mathrm{PKB})$ or Akt, which further negatively regulates FoxO transcriptional factors related to cell cycle and apoptosis [86, 87]. From our point of view, these data are in line with our computational results on the involvement of low PIK3CB in AD pathophysiology via apoptosis and FoxO signaling pathway.

Based on the scatter diagram of the relationship between MM and GS, DEGs strongly interacting with PIK3CB were extracted to construct the global regulatory network for functional annotation, illustrating the value of integrating epigenetic data for understanding complicated mechanisms. The cross-talking pathways of PIK3CB revealed that molecular functions of downregulated PIK3CB in $\mathrm{AD}$ were derived from apoptosis, axon guidance, and FoxO signaling pathway. The vulnerability of such pathways appears to be strikingly apparent at low PIK3CB levels, subsequently contributing to the onset of $\mathrm{AD}$ in a variety of pathogenic mechanisms $[62,72,77,88]$. According to the AUC of $71.7 \%$, low PIK3CB exhibited a good diagnostic performance in $\mathrm{AD}$ prediction, indicating that $\mathrm{PIK} 3 \mathrm{CB}$ may be capable of a genetic risk factor of AD. Consistently, recent evidence from adult drosophila melanogaster suggests that human $\mathrm{A} \beta$ peptide is a candidate site for PI3K phosphorylation, the toxicity of which can be suppressed by co-expression of PI3K [11]. Future in vivo or in vitro experiments are needed to verify the mechanistic pathways of low PIK3CB-mediated AD neurodegeneration that are proposed in this in silico research.

\section{Conclusion}

In aggregate, integrative genomic analysis is an effective approach to uncover pleiotropic roles of PIK3CB underlying $\mathrm{AD}$ development. Our findings lend strong support to the notion that low PIK3CB expression is involved in the pathogenesis of $\mathrm{AD}$ through apoptosis, axon guidance, and FoxO signaling pathway.

\section{Data Availability}

The data supporting the findings of this work are publicly available in GEO database (GSE109887, https://www.ncbi .nlm.nih.gov/gds/?term=GSE109887) and can also be obtainable from the authors upon reasonable request.

\section{Conflicts of Interest}

The authors declare that there is no conflict of interest in this study.

\section{Authors' Contributions}

CSZ, MZ, and ZKZ conceived and designed the study. JB, KXK, RWZ, SSZ, XQZ, and ZKZ performed the experiments and analyzed the data. $M Z$ and $Z K Z$ accomplished the initial draft writing. CSZ, YX, and ZKZ reviewed and edited the paper. All authors read and approved the final version of 
the manuscript. Mei Zhao and Chuansheng Zhao contributed equally to this work.

\section{Acknowledgments}

This research is supported by the National Natural Science Foundation of China (no. 82071467), the Natural Science Foundation of Liaoning Province (no. 20180540150), Innovation Team Support Plan of Universities in Liaoning Province (no. LT2019015), Guidance plan for key research and development plans of Liaoning Province (no. 2019JH8/ 10300002), Discipline Cultivation Support Plan-Stroke Center of China Medical University, Scientific Research Fund of Education Department of Liaoning Province (no. ZF2019012), and Revitalizing Talents Plan of Liaoning Province (Chuansheng Zhao).

\section{References}

[1] K. D. Rynearson, M. Ponnusamy, O. Prikhodko et al., "Preclinical validation of a potent $\gamma$-secretase modulator for Alzheimer's disease prevention," The Journal of Experimental Medicine, vol. 218, 2021.

[2] Z. Zhou, Y. Liang, X. Zhang et al., "Fibrinogen and risk of dementia: a systematic review and meta-analysis," Neuroscience and Biobehavioral Reviews, vol. 112, pp. 353-360, 2020.

[3] S. W. Scheff, S. T. DeKosky, and D. A. Price, "Quantitative assessment of cortical synaptic density in Alzheimer's disease," Neurobiology of Aging, vol. 11, pp. 29-37, 1990.

[4] S. W. Scheff and D. A. Price, "Synapse loss in the temporal lobe in Alzheimer's disease," Annals of Neurology, vol. 33, pp. 190199, 1993.

[5] S. W. Scheff, D. A. Price, F. A. Schmitt, S. T. DeKosky, and E. J. Mufson, "Synaptic alterations in CA1 in mild Alzheimer disease and mild cognitive impairment," Neurology, vol. 68, pp. 1501-1508, 2007.

[6] S. W. Scheff, D. A. Price, F. A. Schmitt, and E. J. Mufson, "Hippocampal synaptic loss in early Alzheimer's disease and mild cognitive impairment," Neurobiology of Aging, vol. 27, pp. 1372-1384, 2006.

[7] A. Serrano-Pozo, M. P. Frosch, E. Masliah, and B. T. Hyman, "Neuropathological alterations in Alzheimer disease," Cold Spring Harbor Perspectives in Medicine, vol. 1, article a006189, 2011.

[8] B. De Strooper, R. Vassar, and T. Golde, "The secretases: enzymes with therapeutic potential in Alzheimer disease," Nature Reviews. Neurology, vol. 6, 2010.

[9] M. P. Kummer and M. T. Heneka, "Truncated and modified amyloid-beta species," Alzheimer's Research \& Therapy, vol. 6, p. 28, 2014.

[10] F. S. Esch, P. S. Keim, E. C. Beattie et al., "Cleavage of amyloid $\beta$ peptide during constitutive processing of its precursor," Science, vol. 248, no. 4959, pp. 1122-1124, 1990.

[11] M. Arnés, N. Romero, S. Casas-Tintó, Á. Acebes, and A. Ferrús, "PI3K activation prevents A $\beta 42$-induced synapse loss and favors insoluble amyloid deposit formation," Molecular Biology of the Cell, vol. 31, pp. 244-260, 2020.

[12] K. Li, Q. Wei, F.-F. Liu et al., "Synaptic dysfunction in Alzheimer's disease: $\mathrm{A} \beta$, tau, and epigenetic alterations," Molecular Neurobiology, vol. 55, pp. 3021-3032, 2018.
[13] D. A. Fruman, R. E. Meyers, and L. C. Cantley, "Phosphoinositide kinases," Annual Review of Biochemistry, vol. 67, pp. 481-507, 1998.

[14] L. C. Cantley, "The phosphoinositide 3-kinase pathway," Science, vol. 296, pp. 1655-1657, 2002.

[15] J. A. Engelman, J. Luo, and L. C. Cantley, "The evolution of phosphatidylinositol 3-kinases as regulators of growth and metabolism," Nature Reviews. Genetics, vol. 7, pp. 606-619, 2006.

[16] D. Wang, W. Zhou, J. Chen, and W. Wei, "Upstream regulators of phosphoinositide 3-kinase and their role in diseases," Journal of Cellular Physiology, 2019.

[17] S. J. Leevers, B. Vanhaesebroeck, and M. D. Waterfield, "Signalling through phosphoinositide 3-kinases: the lipids take centre stage," Current Opinion in Cell Biology, vol. 11, pp. 219-225, 1999.

[18] P.-F. Xu, J.-A. Yang, J.-H. Liu et al., “PI3K $\beta$ inhibitor AZD6482 exerts antiproliferative activity and induces apoptosis in human glioblastoma cells," Oncology Reports, vol. 41, pp. 125-132, 2019.

[19] L. Zhang, Y. Li, Q. Wang et al., “The PI3K subunits, P110 $\alpha$ and P110 $\beta$ are potential targets for overcoming P-gp and BCRPmediated MDR in cancer," Molecular Cancer, vol. 19, p. 10, 2020.

[20] G. Cuesto, L. Enriquez-Barreto, C. Caramés et al., "Phosphoinositide-3-kinase activation controls synaptogenesis and spinogenesis in hippocampal neurons," The Journal of Neuroscience, vol. 31, pp. 2721-2733, 2011.

[21] A. Martín-Peña, A. Acebes, J.-R. Rodríguez et al., “Age-independent synaptogenesis by phosphoinositide 3 kinase," The Journal of Neuroscience, vol. 26, pp. 10199-10208, 2006.

[22] Y. Wang, Y. Lin, L. Wang et al., “TREM2 ameliorates neuroinflammatory response and cognitive impairment via PI3K/ AKT/FoxO3a signaling pathway in Alzheimer's disease mice," Aging (Albany NY), vol. 12, pp. 20862-20879, 2020.

[23] W. Yang, Y. Liu, Q.-Q. Xu, Y.-F. Xian, and Z.-X. Lin, "Sulforaphene ameliorates neuroinflammation and hyperphosphorylated tau protein via regulating the PI3K/Akt/GSK-3 pathway in experimental models of Alzheimer's disease," Oxidative Medicine and Cellular Longevity, vol. 2020, 2020.

[24] Z. Zhou, J. Bai, S. Zhong et al., "Integrative genomic analysis of PPP3R1 in Alzheimer's disease: a potential biomarker for predictive, preventive, and personalized medical approach," The EPMA Journal, vol. 12, pp. 647-658, 2021.

[25] R. Lardenoije, J. A. Y. Roubroeks, E. Pishva et al., “Alzheimer's disease-associated (hydroxy)methylomic changes in the brain and blood," Clinical Epigenetics, vol. 11, p. 164, 2019.

[26] T. Barrett, S. E. Wilhite, P. Ledoux et al., "NCBI GEO: archive for functional genomics data sets-update," Nucleic Acids Research, vol. 41, pp. D991-D995, 2013.

[27] R. Hornung, D. Causeur, C. Bernau, and A.-L. Boulesteix, "Improving cross-study prediction through addon batch effect adjustment or addon normalization," Bioinformatics, vol. 33, pp. 397-404, 2017.

[28] A. Subramanian, P. Tamayo, V. K. Mootha et al., "Gene set enrichment analysis: a knowledge-based approach for interpreting genome-wide expression profiles," Proceedings of the National Academy of Sciences of the United States of America, vol. 102, pp. 15545-15550, 2005.

[29] V. K. Mootha, C. M. Lindgren, K.-F. Eriksson et al., "PGClalpha-responsive genes involved in oxidative 
phosphorylation are coordinately downregulated in human diabetes," Nature Genetics, vol. 34, pp. 267-273, 2003.

[30] A. Reiner, D. Yekutieli, and Y. Benjamini, "Identifying differentially expressed genes using false discovery rate controlling procedures," Bioinformatics, vol. 19, pp. 368-375, 2003.

[31] C. W. Law, Y. Chen, W. Shi, and G. K. Smyth, "Voom: precision weights unlock linear model analysis tools for RNA-seq read counts," Genome Biology, vol. 15, p. R29, 2014.

[32] M. E. Ritchie, B. Phipson, D. Wu et al., "Limma powers differential expression analyses for RNA-sequencing and microarray studies," Nucleic Acids Research, vol. 43, article e47, 2015.

[33] F. N. Soria, A. Pérez-Samartín, A. Martin et al., "Extrasynaptic glutamate release through cystine/glutamate antiporter contributes to ischemic damage," The Journal of Clinical Investigation, vol. 124, pp. 3645-3655, 2014.

[34] S. Horvath, B. Zhang, M. Carlson et al., "Analysis of oncogenic signaling networks in glioblastoma identifies ASPM as a molecular target," Proceedings of the National Academy of Sciences of the United States of America, vol. 103, pp. 1740217407, 2006.

[35] B. Zhang and S. Horvath, "A general framework for weighted gene co-expression network analysis," Statistical Applications in Genetics and Molecular Biology, vol. 4, p. Article17, 2005.

[36] J. A. Miller, R. L. Woltjer, J. M. Goodenbour, S. Horvath, and D. H. Geschwind, "Genes and pathways underlying regional and cell type changes in Alzheimer's disease," Genome Medicine, vol. 5, p. 48, 2013.

[37] P. Langfelder and S. Horvath, "WGCNA: an R package for weighted correlation network analysis," BMC Bioinformatics, vol. 9, no. 1, p. 559, 2008.

[38] Q. Yang, R. Wang, B. Wei et al., "Candidate biomarkers and molecular mechanism investigation for glioblastoma multiforme utilizing WGCNA," BioMed Research International, vol. 2018, Article ID 4246703, 2018.

[39] E. Fredlund, J. Staaf, J. K. Rantala, O. Kallioniemi, A. Borg, and M. Ringnér, "The gene expression landscape of breast cancer is shaped by tumor protein p53 status and epithelialmesenchymal transition," Breast Cancer Research, vol. 14, p. R113, 2012.

[40] D. Szklarczyk, J. H. Morris, H. Cook et al., "The STRING database in 2017: Quality-controlled protein-protein association networks, made broadly accessible," Nucleic Acids Research, vol. 45, pp. D362-D368, 2017.

[41] D. Otasek, J. H. Morris, J. Bouças, A. R. Pico, and B. Demchak, "Cytoscape automation: empowering workflow-based network analysis,” Genome Biology, vol. 20, p. 185, 2019.

[42] P. Shannon, A. Markiel, O. Ozier et al., "Cytoscape: a software environment for integrated models of biomolecular interaction networks," Genome Research, vol. 13, pp. 2498-2504, 2003.

[43] X. Robin, N. Turck, A. Hainard et al., "pROC: an open-source package for R and S+ to analyze and compare ROC curves," BMC Bioinformatics, vol. 12, no. 1, p. 77, 2011.

[44] P. Sonego, A. Kocsor, and S. Pongor, "ROC analysis: applications to the classification of biological sequences and $3 \mathrm{D}$ structures," Briefings in Bioinformatics, vol. 9, pp. 198-209, 2008.

[45] J.-P. Changeux, "Nicotine addiction and nicotinic receptors: lessons from genetically modified mice," Nature Reviews. Neuroscience, vol. 11, pp. 389-401, 2010.
[46] J. R. Monck and J. M. Fernandez, "The exocytotic fusion pore and neurotransmitter release," Neuron, vol. 12, pp. 707-716, 1994.

[47] P. H. Reddy, "A critical assessment of research on neurotransmitters in Alzheimer's disease," Journal of Alzheimer's Disease, vol. 57, pp. 969-974, 2017.

[48] J. J. Renger, C. Egles, and G. Liu, “A developmental switch in neurotransmitter flux enhances synaptic efficacy by affecting AMPA receptor activation," Neuron, vol. 29, pp. 469-484, 2001.

[49] N. Rama, D. Goldschneider, V. Corset, J. Lambert, L. Pays, and P. Mehlen, "Amyloid precursor protein regulates netrin-1mediated commissural axon outgrowth," The Journal of Biological Chemistry, vol. 287, pp. 30014-30023, 2012.

[50] S. L. Sabo, A. F. Ikin, J. D. Buxbaum, and P. Greengard, "The amyloid precursor protein and its regulatory protein, FE65, in growth cones and synapses in vitro and in vivo," The Journal of Neuroscience, vol. 23, pp. 5407-5415, 2003.

[51] A. Soldano and B. A. Hassan, "Beyond pathology: APP, brain development and Alzheimer's disease," Current Opinion in Neurobiology, vol. 27, pp. 61-67, 2014.

[52] I. Västrik, B. J. Eickholt, F. S. Walsh, A. Ridley, and P. Doherty, "Sema3A-induced growth-cone collapse is mediated by Rac1 amino acids 17-32," Current Biology, vol. 9, pp. 991-998, 1999.

[53] N. Gakhar-Koppole, P. Hundeshagen, C. Mandl et al., "Activity requires soluble amyloid precursor protein alpha to promote neurite outgrowth in neural stem cell-derived neurons via activation of the MAPK pathway," The European Journal of Neuroscience, vol. 28, pp. 871-882, 2008.

[54] S. Chasseigneaux and B. Allinquant, "Functions of A $\beta$, sAPP $\alpha$ and $\operatorname{sAPP} \beta$ : similarities and differences," Journal of Neurochemistry, 2012, 120 Suppl 1.

[55] S. Chasseigneaux, L. Dinc, C. Rose et al., "Secreted amyloid precursor protein $\beta$ and secreted amyloid precursor protein $\alpha$ induce axon outgrowth in vitro through Egr1 signaling pathway," PLoS One, vol. 6, article e16301, 2011.

[56] K. K. Freude, M. Penjwini, J. L. Davis, F. M. LaFerla, and M. Blurton-Jones, "Soluble amyloid precursor protein induces rapid neural differentiation of human embryonic stem cells," The Journal of Biological Chemistry, vol. 286, pp. 2426424274, 2011.

[57] T. Kuboyama, Y.-A. Lee, H. Nishiko, and C. Tohda, "Inhibition of clathrin-mediated endocytosis prevents amyloid $\beta$ induced axonal damage," Neurobiology of Aging, vol. 36, pp. 1808-1819, 2015.

[58] L. Zhang, Z. Qi, J. Li et al., "Roles and mechanisms of axonguidance molecules in Alzheimer's disease," Molecular Neurobiology, 2021.

[59] K. Waite and B. J. Eickholt, "The neurodevelopmental implications of PI3K signaling," Current Topics in Microbiology and Immunology, vol. 346, pp. 245-265, 2010.

[60] F.-Q. Zhou, J. Zhou, S. Dedhar, Y.-H. Wu, and W. D. Snider, "NGF-induced axon growth is mediated by localized inactivation of GSK-3beta and functions of the microtubule plus end binding protein APC,” Neuron, vol. 42, pp. 897-912, 2004.

[61] W. Lee, C. W. Frank, and J. Park, "Directed axonal outgrowth using a propagating gradient of IGF-1," Advanced Materials, vol. 26, pp. 4936-4940, 2014.

[62] L. Laurino, X. X. Wang, B. A. de la Houssaye et al., "PI3K activation by IGF-1 is essential for the regulation of membrane 
expansion at the nerve growth cone," Journal of Cell Science, vol. 118, pp. 3653-3662, 2005.

[63] L. Sosa, S. Dupraz, L. Laurino et al., "IGF-1 receptor is essential for the establishment of hippocampal neuronal polarity," Nature Neuroscience, vol. 9, pp. 993-995, 2006.

[64] J. K. Atwal, K. K. Singh, M. Tessier-Lavigne, F. D. Miller, and D. R. Kaplan, "Semaphorin 3F antagonizes neurotrophininduced phosphatidylinositol 3-kinase and mitogen-activated protein kinase kinase signaling: a mechanism for growth cone collapse," The Journal of Neuroscience, vol. 23, pp. 7602-7609, 2003.

[65] Y. Ito, I. Oinuma, H. Katoh, K. Kaibuchi, and M. Negishi, "Sema4D/plexin-B1 activates GSK-3beta through R-Ras GAP activity, inducing growth cone collapse," EMBO Reports, vol. 7, pp. 704-709, 2006.

[66] G. J. Colurso, J. E. Nilson, and L. G. Vervoort, "Quantitative assessment of DNA fragmentation and beta-amyloid deposition in insular cortex and midfrontal gyrus from patients with Alzheimer's disease," Life Sciences, vol. 73, pp. 1795-1803, 2003.

[67] O. Levi and D. M. Michaelson, "Environmental enrichment stimulates neurogenesis in apolipoprotein E3 and neuronal apoptosis in apolipoprotein E4 transgenic mice," Journal of Neurochemistry, vol. 100, pp. 202-210, 2007.

[68] C. Frey, A. Bonert, T. Kratzsch et al., "Apolipoprotein E epsilon 4 is associated with an increased vulnerability to cell death in Alzheimer's disease," Journal of Neural Transmission (Vienna), vol. 113, pp. 1753-1761, 2006.

[69] Z.-S. Ji, K. Müllendorff, I. H. Cheng, R. D. Miranda, Y. Huang, and R. W. Mahley, "Reactivity of apolipoprotein E4 and amyloid beta peptide: lysosomal stability and neurodegeneration," The Journal of Biological Chemistry, vol. 281, pp. 2683-2692, 2006.

[70] K. Takuma, J. Yao, J. Huang et al., “ABAD enhances Abetainduced cell stress via mitochondrial dysfunction," The FASEB Journal, vol. 19, pp. 597-598, 2005.

[71] E. Ferreiro, C. R. Oliveira, and C. M. F. Pereira, “The release of calcium from the endoplasmic reticulum induced by amyloidbeta and prion peptides activates the mitochondrial apoptotic pathway," Neurobiology of Disease, vol. 30, pp. 331-342, 2008.

[72] F. Yan, X. Tan, W. Wan et al., "ErbB4 protects against neuronal apoptosis via activation of YAP/PIK3CB signaling pathway in a rat model of subarachnoid hemorrhage," Experimental Neurology, vol. 297, 2017.

[73] Z. Zhang, L. Zhang, B. Wang et al., "MiR-337-3p suppresses proliferation of epithelial ovarian cancer by targeting PIK3CA and PIK3CB," Cancer Letters, vol. 469, pp. 54-67, 2020.

[74] B. Cen, Y. Wei, W. Huang et al., "An efficient bivalent cyclic RGD-PIK3CB siRNA conjugate for specific targeted therapy against glioblastoma in vitro and in vivo," Molecular Therapy-Nucleic Acids, vol. 13, pp. 220-232, 2018.

[75] K. J. Pridham, L. Le, S. Guo et al., "PIK3CB/p110 $\beta$ is a selective survival factor for glioblastoma," Neuro-Oncology, vol. 20, pp. 494-505, 2018.

[76] A. D. Whale, L. Colman, L. Lensun, H. L. Rogers, and S. J. Shuttleworth, "Functional characterization of a novel somatic oncogenic mutation of_PIK3CB_," Signal Transduction and Targeted Therapy, vol. 2, no. 1, p. 17063, 2017.

[77] K. N. Manolopoulos, L. O. Klotz, P. Korsten, S. R. Bornstein, and A. Barthel, "Linking Alzheimer's disease to insulin resis- tance: the FoxO response to oxidative stress," Molecular Psychiatry, vol. 15, pp. 1046-1052, 2010.

[78] R. Pardeshi, N. Bolshette, K. Gadhave et al., "Insulin signaling: an opportunistic target to minify the risk of Alzheimer's disease," Psychoneuroendocrinology, vol. 83, pp. 159-171, 2017.

[79] D. H. Kim, M. H. Park, E. K. Lee et al., "The roles of FoxOs in modulation of aging by calorie restriction," Biogerontology, vol. 16, 2015.

[80] J. Buteau and D. Accili, "Regulation of pancreatic?-cell function by the forkhead protein FoxO1," Diabetes, Obesity \& Metabolism, vol. 9, Suppl 2, pp. 140-146, 2007.

[81] H.-C. Chiang, L. Wang, Z. Xie, A. Yau, and Y. Zhong, "PI3 kinase signaling is involved in Abeta-induced memory loss in drosophila," Proceedings of the National Academy of Sciences of the United States of America, vol. 107, pp. 7060-7065, 2010.

[82] R. Sandhir and S. Gupta, "Molecular and biochemical trajectories from diabetes to Alzheimer's disease: a critical appraisal," Journal of Diabetes, vol. 6, no. 12, pp. 1223-1242, 2015.

[83] A.-L. Hsu, C. T. Murphy, and C. Kenyon, "Regulation of aging and age-related disease by DAF-16 and heat-shock factor," Science, vol. 300, pp. 1142-1145, 2003.

[84] M. Jazvinšćak Jembrek, P. R. Hof, and G. Šimić, “Ceramides in Alzheimer's disease: key mediators of neuronal apoptosis induced by oxidative stress and $\mathrm{A} \beta$ accumulation," Oxidative Medicine and Cellular Longevity, vol. 2015, Article ID 346783, 2015.

[85] W. W. Smith, D. D. Norton, M. Gorospe et al., "Phosphorylation of p66Shc and forkhead proteins mediates Abeta toxicity," The Journal of Cell Biology, vol. 169, pp. 331-339, 2005.

[86] A. Brunet, A. Bonni, M. J. Zigmond et al., "Akt promotes cell survival by phosphorylating and inhibiting a Forkhead transcription factor," Cell, vol. 96, pp. 857-868, 1999.

[87] Y. Furukawa-Hibi, Y. Kobayashi, C. Chen, and N. Motoyama, "FOXO transcription factors in cell-cycle regulation and the response to oxidative stress," Antioxidants \& Redox Signaling, vol. 7, pp. 752-760, 2005.

[88] H.-Z. Long, Y. Cheng, Z.-W. Zhou, H.-Y. Luo, D.-D. Wen, and L.-C. Gao, "PI3K/AKT signal pathway: a target of natural products in the prevention and treatment of Alzheimer's disease and Parkinson's disease," Frontiers in Pharmacology, vol. 12, article 648636, 2021. 